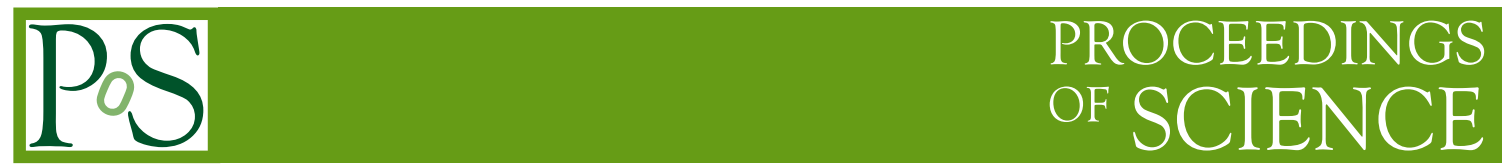

\title{
Non-commutative geometry from large $N$ gauge theories
}

\author{
John Iliopoulos*t \\ École Normale Supérieure, 75005 Paris, France \\ E-mail: ilio@lpt.ens.fr
}

We show that a classical $S U(N)$ gauge theory on a $d$-dimensional space-time can be reformulated as a theory on $d+2$-dimensions with the extra two dimensions describing a fuzzy closed surface. The coordinates of this surface satisfy a Heisenberg algebra with the deformation parameter proportional to $1 / N$.

Corfu Summer Institute 2016 "School and Workshops on Elementary Particle Physics and Gravity" 31 August - 23 September, 2016

Corfu, Greece

\footnotetext{
* Speaker.

${ }^{\dagger}$ Talk presented at the Ioannis BAKAS memorial Conference, Ringberg, November 2016.
} 


\section{Forward}

I met Yannis for the first time in 1983. I was visiting Imperial College for a seminar and the late Tom Kibble, then Chairman of the Department, recommended to me a few bright Greek graduate students in Theoretical Physics. Yannis was among them. During the following years I had the occasion to confirm this opinion. We met often in École Normale, at CERN as well as in Conferences and summer schools and Yannis soon established himself as a leading scientist in our field. In recent years I discovered another facet of his personality: his profound sense of commitment and responsibility. He was deeply concerned with the harsh conditions of young scientists working in Greece under the current crisis and he decided to help. He convinced the authorities of his native town, Kalamata, to establish a special prize, "the Messinian Prize for Excellency", and asked me to join this effort. The first such prizes were awarded in October 2015 to two young high energy physicists, one experimentalist and one theorist, by our distinguished guests Jim Cronin and David Gross. I promised to continue this effort, but it won't be easy without Yannis. In his quiet and simple manner he was able to inspire, convince and mobilise.

These notes give a summary of a discussion we had together in the first days of August 2016, just two days before he entered the Hospital for the last time. It was probably Yannis' last scientific discussion. He was optimistic and confident that his health problems would be soon over and we promised to meet again in a few weeks to continue our discussion. So, I felt a great shock when I heard the news of his death. I sent a message to École Normale and I remember a young colleague answering that this was impossible: just very recently Yannis had published two papers in the ArXiv. He was right. Bakas will remain in the scientific literature, but we, who have had the privilege to know him, will always remember Yannis.

\section{Introduction}

I am reporting here on some on-going work done in collaboration with Prof. Manolis Floratos. The main results have already been published,[1] but there are some open problems remaining.

There may be several motivations to study a quantum theory in a space with non-commutative geometry. The first proposal goes back to W. Heisenberg who, in a letter to Peierls in 1930[2], suggested that non-commutativity among space coordinates could eliminate the short distance singularities. He tried to convince Peierls and Pauli to work on this problem, but, apparently, Pauli did not think much of the idea. ${ }^{1}$ He talked instead to Oppenheimer[4] who gave it as a problem to his $\mathrm{PhD}$ student H.S. Snyder. Snyder published a thorough study of it in 1947[5], but there was no follow up for many years. Snyder himself left non-commutative geometry and had a successful career as an accelerator engineer[6]. In fact, as history evolved, Pauli was probably right. The motivation based on short distance singularities did not prove fruitful for elementary particle physics. With the development of the renormalisation programme in the framework of quantum field theories, the problem of ultraviolet divergences took a completely different turn. While a space cut-off makes all theories finite, the renormalisation programme applies to very few and very specific field theories. It is a most remarkable fact that they are precisely the ones chosen by Nature. It is not finiteness but rather lack of sensitivity to unknown physics at very short distances that turned out to be the

\footnotetext{
${ }^{1}$ In a letter to Bohr he commented : “....it seems to be a failure for reasons of physics.”[3]
} 
important criterion. The geometry of physical space may still produce an ultraviolet cut-off, but its presence is not relevant for the calculation of physical processes among elementary particles.

However, almost at the same time, a new motivation for studying theories in a non-commutative space appeared, although only recently it was fully appreciated. In 1930 L.D. Landau[7] solved the problem of the motion of an electron in an external constant magnetic field and, besides computing the energy levels, the so-called "Landau levels", he showed that the components of the velocity operator of the electron do not commute. A simple way to visualise this result is to think of the classical case where the electron follows a spiral trajectory whose projection on a plane perpendicular to the field is a circle. In Landau's quantum mechanical solution the centre's coordinates are:

$$
x_{c}=\frac{c p_{y}}{e H}+x \quad ; \quad y_{c}=-\frac{c p_{x}}{e H}
$$

which shows that the two coordinates do not commute. The magnetic field has induced a noncommutative structure on space itself. Following Heisenberg's suggestion, R. Peierls[8] showed that, at least the lowest Landau level, can be obtained by using this space non-commutativity. Since the presence of non-vanishing magnetic-type external fields is a common feature in many modern supergravity and string models, the study of field theories formulated on spaces with noncommutative geometry[9] has become quite fashionable. A new element was added a few years ago with the work of N. Seiberg and E. Witten[10] who showed the existence of a map between gauge theories formulated in spaces with commuting and non-commuting coordinates. A somehow independent line of approach has been initiated by A. Connes[9][11] and co-workers and aims at constructing a gauge theory with spontaneously broken symmetry using the techniques of noncommutative geometry. The result which relates the symmetry breaking parameter to the distance between different branes has been first obtained in this approach. There have been many review articles, both from the mathematics as well as the physics point of view, and an incomplete list is given in reference[12]. The more recent advances have been presented in several CORFU meetings. Here I want to mention a different but related motivation which comes from $S U(N)$ gauge theories at large $N$ and matrix models.

\section{Some elementary formulae}

Space non-commutativity means that we have a commutation relation of the form:

$$
\left[x_{\mu}, x_{v}\right]=i \theta_{\mu \nu}
$$

In the simplest case, the one we will consider in this talk, $\theta$ is constant (canonical, or Heisenberg case). Other choices have also been considered: $\left[x_{\mu}, x_{v}\right]=i F_{\mu v}^{\rho} x_{\rho}$ (Lie algebra case, the one analysed by Snyder), or $x_{\mu} x_{v}=q^{-1} R_{\mu \nu}^{\rho \sigma} x_{\rho} x_{\sigma}$, (sometimes called quantum space case).

We also define the derivative:

$$
\partial^{\mu} x_{v}=\delta_{v}^{\mu} \Rightarrow\left[x_{\mu}, f(x)\right]=i \theta_{\mu \nu} \partial^{v} f(x)
$$

and $\mathrm{a} *$ product which is formally given by:

$$
f * g=\left.e^{\frac{i}{2} \frac{\partial}{x_{\mu}} \theta_{\mu v} \frac{\partial}{y_{v}}} f(x) g(y)\right|_{x=y}
$$


All computations can be viewed as expansions in $\theta$, which, in the Landau paradigm, is proportional to the inverse of the external magnetic field. It is not clear whether any more efficient computational tools can be developed which would take full advantage of the non-commutative nature of space and this seems to be a severe limitation of the power of the approach. We do not have a quantum field theory in a space with non-commutative geometry which is not an expansion around ordinary space. This may be the reason why most formulations of gauge theories seem to be limited to the classical theory and it is not easy to extend to them the BRST symmetry.

The main remark which underlines our approach is the following: Let $\phi^{i}(x) i=1, \ldots, N N$ $\rightarrow \infty$ be an $N$-component field defined in a $d$-dimensional space. At large $N$ we can write $\phi^{i}(x)$ $\rightarrow \phi(\sigma, x) 0 \leq \sigma \leq 2 \pi$, i.e. we can consider $\phi^{i}(x)$ as the Fourier components of a field in $d+1$ dimensions with the extra dimension being a circle. In this case we have:

$$
\sum_{i=1}^{\infty} \phi^{i}(x) \phi^{i}(x) \rightarrow \int_{0}^{2 \pi} \mathrm{d} \sigma(\phi(\sigma, x))^{2}
$$

However, the interaction term will be non local in $\sigma$ :

$$
\phi^{4} \rightarrow\left(\int_{0}^{2 \pi} \mathrm{d} \sigma(\phi(\sigma, x))^{2}\right)^{2}
$$

The crucial remark is that, for a Yang-Mills field $A_{\mu}(x)$ belonging to the adjoint representation of an $S U(N)$ group, the resulting expression at large $N$ is local[1]. Written explicitly we have:

Given an $S U(N)$ Yang-Mills theory in a $d$-dimensional space $A_{\mu}(x)=A_{\mu}^{a}(x) t_{a}$, there exists a reformulation in $d+2$ dimensions $A_{\mu}(x) \rightarrow \mathscr{A}_{\mu}\left(x, \sigma_{1}, \sigma_{2}\right)$ and $F_{\mu v}(x) \rightarrow \mathscr{F}_{\mu v}\left(x, \sigma_{1}, \sigma_{2}\right)$ with $\sigma_{1}$ and $\sigma_{2}$ appropriately chosen coordinates on a compact 2-dimensional surface, such that, at $N \rightarrow \infty$, the matrix commutators become the usual Poisson brackets with respect to $\sigma_{1}$ and $\sigma_{2}$.

$$
\begin{aligned}
{\left[A_{\mu}(x), A_{v}(x)\right] } & \rightarrow\left\{\mathscr{A}_{\mu}\left(x, \sigma_{1}, \sigma_{2}\right), \mathscr{A}_{v}\left(x, \sigma_{1}, \sigma_{2}\right)\right\} \\
{\left[A_{\mu}(x), W(x)\right] } & \rightarrow\left\{\mathscr{A}_{\mu}\left(x, \sigma_{1}, \sigma_{2}\right), \mathscr{W}\left(x, \sigma_{1}, \sigma_{2}\right)\right\}
\end{aligned}
$$

where $W$ is an element of the gauge group. We can show that the Yang-Mills action becomes:

$$
\int \mathrm{d}^{4} x \operatorname{Tr}\left(F_{\mu v}(x) F^{\mu v}(x)\right) \rightarrow \int \mathrm{d}^{4} x \mathrm{~d} \sigma_{1} \mathrm{~d} \sigma_{2} \mathscr{F}_{\mu v}\left(x, \sigma_{1}, \sigma_{2}\right) \mathscr{F}^{\mu v}\left(x, \sigma_{1}, \sigma_{2}\right)
$$

The $S U(N)$ gauge invariance has become invariance under area preserving diffeomorphisms of the 2-dimensional surface spanned by $\sigma_{1}$ and $\sigma_{2}$. The proof of this statement is essentially algebraic. A direct way[13] is to prove that at the limit $N \rightarrow \infty$ the $S U(N)$ structure constants, appropriately rescaled, go to those of [SDiff 2$]$. We can also show it explicitly for the sphere[1] and the torus[1][14]

A final remark: The equivalence (3.7) is established for the classical theories. To go to the quantum theory we should first find a suitable gauge and this can be done. But then we are facing a second problem: The quadratic part of the new 6-dimensional action has no derivatives with respect to the variables $\sigma_{1}$ and $\sigma_{2}$. As a result, the perturbation expansion cannot be defined. This is not surprising. In proving (3.7) we have not imposed 't Hooft's rescaling condition in which $g^{2} N$ is kept fixed and we recover, already at lowest order, the infinite number of graphs. It is possible, 
although we have no explicit proof, that we can absorb these divergences in a clever renormalisation scheme, but it is not clear whether any new insight can be obtained this way. The 4-dimensional theory we started from is renormalisable for any finite $N$. A different approach would be to expand around a non-trivial solution which, hopefully, captures part of the non-perturbative dynamics of the Yang-Mills theory. Such a "master field" has not yet been found.

\section{Finite $N$ and non-commutative geometry}

The previous results were valid at $N \rightarrow \infty$. In this section I want to point out that we can extend them to any value of $N$ with the price of introducing non-commutative geometry for the extra 2-dimensional space[1]. I will sketch the proof for a "fuzzy" sphere and a "fuzzy" torus.

\subsection{A fuzzy sphere}

For the classical sphere a convenient choice of coordinates is given by the usual angles $\theta$ and $\phi$. We can write $x_{1}=\cos \phi \sin \theta, x_{2}=\sin \phi \sin \theta$ and $x_{3}=\cos \theta$. The corresponding spherical harmonics are given by:

$$
Y_{l, m}(\theta, \phi)=\sum_{\substack{i_{k}=1,2,3 \\ k=1, \ldots, l}} \alpha_{i_{1} \ldots i_{l}}^{(m)} x_{i_{1}} \ldots x_{i_{l}}
$$

where $\alpha_{i_{1} \ldots i_{l}}^{(m)}$ is a symmetric and traceless tensor. For fixed $l$ there are $2 l+1$ linearly independent tensors $\alpha_{i_{1} \ldots i_{l}}^{(m)}, m=-l, \ldots, l$.

We choose, inside $S U(N)$, an $S U(2)$ subgroup $^{2}$ whose generators we call $S_{i}$. They satisfy the commutation relations: $\left[S_{i}, S_{j}\right]=\mathrm{i} \varepsilon_{i j k} S_{k}$. We can use them as a basis to build the $N^{2}-1$ generators of $S U(N)$ in the fundamental representation:

$$
S_{l, m}^{(N)}=\sum_{\substack{i_{k}=1,2,3 \\ k=1, \ldots, l}} \alpha_{i_{1} \ldots i_{l}}^{(m)} S_{i_{1} \ldots} \ldots S_{i_{l}} \Rightarrow\left[S_{l, m}^{(N)}, S_{l^{\prime}, m^{\prime}}^{(N)}\right]=\mathrm{i} f_{l, m, l^{\prime}, m^{\prime}}^{(N) l^{\prime \prime}, m^{\prime \prime}} S_{l^{\prime \prime}, m^{\prime \prime}}^{(N)}
$$

where the constants $f^{(N)}$ are the $S U(N)$ structure constants in a somehow unusual notation. It is now clear that the three $S U(2)$ generators $S_{i}$, rescaled by a factor proportional to $1 / N$, will have well-defined limits as $N$ goes to infinity:

$$
S_{i} \rightarrow T_{i}=\frac{2}{N} S_{i} \text { implies }\left[T_{i}, T_{j}\right]=\frac{2 \mathrm{i}}{N} \varepsilon_{i j k} T_{k} \text { and } T^{2}=T_{1}^{2}+T_{2}^{2}+T_{3}^{2}=1-\frac{1}{N^{2}}
$$

In other words: under the norm $\|x\|^{2}=\operatorname{Tr} x^{2}$, the limits as $N$ goes to infinity of the generators $T_{i}$ are three objects $x_{i}$ which commute and are constrained by $x_{1}^{2}+x_{2}^{2}+x_{3}^{2}=1$. This in turn shows that the classical Yang-Mills theory becomes the theory invariant under area preserving diffeomorphisms of equation (3.7) with the closed surface being a sphere $S^{2}$.

So much for the large $N$ limit. For any value of $N$, we can parametrise the three operators $T_{i}$ in terms of two operators, $z_{1}$ and $z_{2}$ as follows: ${ }^{3}$

\footnotetext{
${ }^{2}$ To be precise, it must be the principal $S U(2)$ subgroup[15]. In practice this means that we must choose a suitable basis in the $S U(N)$ Lie algebra such that the generators of the chosen $S U(2)$ are represented in the standard way by $N \times N$ matrices forming an irreducible representation.

${ }^{3} \mathrm{~A}$ similar parametrisation has been used by T. Holstein and H. Primakoff in terms of creation and annihilation operators[16].
} 


$$
\begin{gathered}
T_{+}=T_{1}+\mathrm{i} T_{2}=e^{\frac{\mathrm{i} z_{1}}{2}}\left(1-z_{2}^{2}\right)^{\frac{1}{2}} e^{\frac{\mathrm{i} z_{1}}{2}} \\
T_{-}=T_{1}-\mathrm{i} T_{2}=e^{-\frac{\mathrm{i}_{1}}{2}}\left(1-z_{2}^{2}\right)^{\frac{1}{2}} e^{-\frac{\mathrm{i} z_{1}}{2}} \\
T_{3}=z_{2}
\end{gathered}
$$

Then it is straightforward algebra[1] to prove the following algebraic statement:

$$
\left[z_{1}, z_{2}\right]=\frac{2 i}{N} \Leftrightarrow\left[T_{i}, T_{j}\right]=\frac{2 \mathrm{i}}{N} \varepsilon_{i j k} T_{k}
$$

in other words, if $z_{1}$ and $z_{2}$ satisfy the Heisenberg algebra, the operators $T_{i}$ satisfy the $S U(2)$ algebra and the opposite is also true, the $S U(2)$ algebra for the operators $T_{i}$ imply the Heisenberg algebra among $z_{1}$ and $z_{2}$. From that point we can go on and show a formal equivalence:

Given an $S U(N)$ Yang-Mills theory in a $d$-dimensional space, there exists a reformulation in $d+2$ dimensions in which

$$
A_{\mu}(x) \rightarrow \mathscr{A}_{\mu}\left(x, z_{1}, z_{2}\right) \quad F_{\mu \nu}(x) \rightarrow \mathscr{F}_{\mu \nu}\left(x, z_{1}, z_{2}\right) \text { and }\left[z_{1}, z_{2}\right]=\frac{2 i}{N}
$$

such that

$$
\begin{aligned}
{\left[A_{\mu}(x), A_{v}(x)\right] } & \rightarrow\left\{\mathscr{A}_{\mu}\left(x, z_{1}, z_{2}\right), \mathscr{A}_{v}\left(x, z_{1}, z_{2}\right)\right\}_{\text {Moyal }} \\
{\left[A_{\mu}(x), W(x)\right] } & \rightarrow\left\{\mathscr{A}_{\mu}\left(x, z_{1}, z_{2}\right), \mathscr{W}\left(x, z_{1}, z_{2}\right)\right\}_{\text {Moyal }}
\end{aligned}
$$

where the brackets are the symmetrised Moyal brackets[17] with respect to the operators $z_{1}$ and $z_{2}$ and the action becomes:

$$
\int \mathrm{d}^{4} x \operatorname{Tr}\left(F_{\mu v}(x) F^{\mu v}(x)\right) \rightarrow \int \mathrm{d}^{4} x \mathrm{~d} z_{1} \mathrm{~d} z_{2} \mathscr{F}_{\mu v}\left(x, z_{1}, z_{2}\right) * \mathscr{F}^{\mu v}\left(x, z_{1}, z_{2}\right)
$$

with an appropriately defined $*$-product.

\subsection{A fuzzy torus}

The case of the fuzzy torus is even simpler. For the sphere we had isolated inside $S U(N)$ an $S U(2)$ subgroup and express all the $S U(N)$ generators in terms of the three generators of $S U(2)$. For the torus we isolate a quantum $U(1) \times U(1)$. Let us take first $N$ odd (a similar construction applies to $N$ even) and let $\omega$ be the $N$ th root of unity: $\omega=\mathrm{e}^{4 \pi \mathrm{i} / N}$. We define the two matrices:

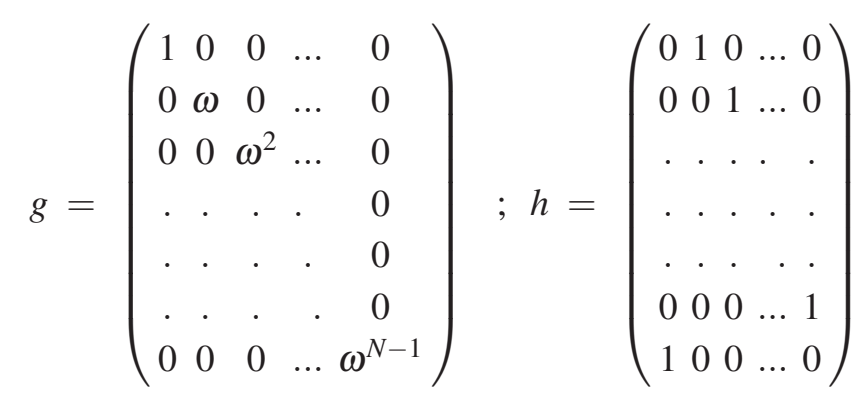

They satisfy quantum group commutation relations:

$$
g^{N}=h^{N}=1 \quad ; \quad h g=\omega g h
$$


We can use the integer $\bmod N$ powers of these matrices to express the $S U(N)$ generators:

$$
\begin{gathered}
S_{m_{1}, m_{2}}=\omega^{m_{1} m_{2} / 2} g^{m_{1}} h^{m_{2}} ; S_{m_{1}, m_{2}}^{\dagger}=S_{-m_{1},-m_{2}} \\
{\left[S_{\mathbf{m}}, S_{\mathbf{n}}\right]=2 \mathrm{i} \sin \left(\frac{2 \pi}{N} \mathbf{m} \times \mathbf{n}\right) S_{\mathbf{m}+\mathbf{n}}}
\end{gathered}
$$

with $\mathbf{n}=\left(n_{1}, n_{2}\right)$ and $\mathbf{n} \times \mathbf{m}=n_{1} m_{2}-m_{1} n_{2}$. We can show[18] that the algebra (4.11) is indeed equivalent to that of $S U(N)$ and at the limit $N \rightarrow \infty$ it becomes the algebra of the area preserving diffeomorphisms of a 2-dimensional torus. This connection between $S U(N)$ and [SDiff(T2)] can be made explicit by choosing a pair of variables forming local symplectic coordinates on the torus, for example, the angles $z_{1}$ and $z_{2}$ of the two circles, and expanding all functions on the torus on the basis of the eigenfunctions of the laplacian:

$$
h_{n_{1}, n_{2}}=\exp \left(\mathrm{i} n_{1} z_{1}+2 \pi \mathrm{i} n_{2} z_{2}\right) \quad n_{1}, n_{2} \in \mathbb{Z}
$$

Here we are interested in the fuzzy torus, so we endow $z_{1}$ and $z_{2}$ with the commutation relations of the Heisenberg algebra (4.4). If we define the corresponding group elements $h$ and $g$, by:

$$
h=\mathrm{e}^{\mathrm{i} z_{1}} \quad g=\mathrm{e}^{-2 \mathrm{i} \pi z_{2}}
$$

we can prove again the equivalence:

$$
\left[z_{1}, z_{2}\right]=\frac{2 i}{N} \Leftrightarrow h g=\omega g h
$$

for the set of group elements $h^{n_{1}}$ and $g^{n_{2}}$ with $n_{1}$ and $n_{2}$ integers $\bmod N$. Note that the later imply the algebra of $S U(N)$. The generators of the Heisenberg algebra $z_{i}$ and the group elements $h$ and $g$ are infinite dimensional operators, but we can represent the $S U(N)$ algebra by the finite dimensional ones (4.8) and (4.10). They form a discrete subgroup of the Heisenberg group and they have been used to construct quantum mechanics on a discrete phase space[19]. In this case, we can define two new operators $\hat{q}$ and $\hat{p}$, the first being the position operator on the discrete configuration space and the second its finite Fourier transform. They can be represented by $N \times N$ matrices, but, obviously, they do not satisfy anymore the Heisenberg algebra[20].

As we found for the sphere, the Moyal bracket can be defined by symmetrising in $z_{1}$ and $z_{2}$, in which case only odd powers of $1 / N$ appear. The *-product can be written as

$$
f(z) * g(z)=\left.\exp \left(\mathrm{i} \xi \varepsilon_{i j} \partial_{z}^{i} \partial_{w}^{j}\right) f(z) g(w)\right|_{w=z}
$$

with $z=\left(z_{1}, z_{2}\right)$ and $\xi=2 / N$. The Yang-Mills action can be written again in the form of equations (4.5) and (4.7) and, as before for the sphere, this equivalence is exact at any order in the $1 / N$ expansion.

\section{Conclusions and remarks}

Let me finish with some randomly chosen remarks.

- At the formal level, all the previous expressions do not require $N$ to be an integer. It could be any, even complex, number. So this writing offers a way to define the theory for any $N$. Could this offer a new insight? 
- The non-commutative geometry we found here appears to be only formal, it translates the fact that the gauge fields in a Yang-Mills theory are matrix valued. It is a different, but formally equivalent way to write the theory.

- The approach initiated by A. Connes and co-workers[9][11] was more ambitious. It gave a new qualitative insight by associating the scale of spontaneous symmetry breaking to the distance between different branes, a picture which has emerged also in string theories. However, no satisfactory quantitative predictions were obtained. We know now that the seventeen parameters of the Standard Model form an irreducible set from the renormalisation group point of view and, as long as we stay with the usual perturbation expansion in commuting space, no relation among them can be stable.

- This brings me to the last point which applies to all the formulations I have seen so far. We do not have a correct quantum field theory defined directly in a space with non-commutative geometry without referring to the limiting commuting space. All formulations, one way or another, amount to expansions in the non-comutative parameter. This way we cannot expect to capture the non-perturbative aspects of gauge theories, which are precisely the ones we want. It is the kind of questions I would have liked to discuss with Yannis.

\section{References}

[1] E.G. Floratos and J. Iliopoulos, Phys. Lett. B201, 237 (1988); E.G. Floratos, J. Iliopoulos and G. Tiktopoulos, Phys. Lett. B217, 285 (1989); E.G. Floratos and J. Iliopoulos, Phys. Lett. B632, 566 (2006)

[2] Letter of Heisenberg to Peierls (1930), Wolfgang Pauli, Scientific Correspondence, Vol. II, p. 15. (Ed. Karl von Meyenn, Springer-Verlag (1985))

[3] Letter of Pauli to Bohr (1947), Wolfgang Pauli, Scientific Correspondence, Vol. II, p. 414. (Ed. Karl von Meyenn, Springer-Verlag (1985))

[4] Letter of Pauli to Oppenheimer (1946), Wolfgang Pauli, Scientific Correspondence, Vol. III, p. 380. (Ed. Karl von Meyenn, Springer-Verlag (1993))

[5] H.S. Snyder, Phys. Rev. 71, 38 (1947). Apparently Snyder had completed this work as early as 1943.

[6] E.D. Courant, M.S. Livingston and H.S. Snyder, Phys. Rev. 88, 1190 (1952)

[7] L.D. Landau, Z. Phys. 64, 629 (1930)

[8] R. Peierls, Z. Phys. 80, 763 (1933)

[9] For a review, see A. Connes, "Non-Commutative Geometry and Physics", Les Houches summer school, 1992

[10] N. Seiberg and E. Witten, JHEP 09, 032 (1999)

[11] See, for example, A.H. Chamseddine and A. Connes, Fortsch. Phys. 58, 553 (2010); A.H. Chamseddine, A. Connes and V. Mukhanov, JHEP 1412, 098 (2014)

[12] For some reviews see: J. Wess, "Non abelian gauge theories on non-commutative spaces", Proceedings, SUSY02; J. Madore, "An Introduction to non-Commutative Differential Geometry and Physical applications”, Cambridge, UK, University Press, 2000; M. R. Douglas and N. A. Nekrasov, Rev. Mod. Phys. 73, 977 (2001); J. Wess, "Gauge theories beyond gauge theories”, Fortsch. Phys. 49, 
377 (2001); J. Wess, “Gauge theories on non-commutative spaces”, Lect. Notes Phys. 616, 320 (2003); R. Jackiw, "Physical instances of noncommuting coordinates", hep-th/0110057; B. Zumino, "Deformation quantisation of nonabelian gauge groups", Proceedings, SUGRA 20; R.J. Szabo, Phys. Rep. 378, 207 (2003)

[13] J. Hoppe, MIT-PhD Thesis, 1982

[14] D. B. Fairlie, P. Fletcher and C. K. Zachos, Phys. Lett. B218, 203 (1989)

[15] B. Konstant, Am. Journ. of Math. 81, 4, 973 (1959)

[16] T. Holstein and H. Primakoff, Phys. Rev. 58, 1098 (1940)

[17] J. Moyal, Proc. Camb. Phil. Soc. 45, 99 (1949)

[18] D. B. Fairlie and C. K. Zachos, Phys. Lett. B224, 101 (1989)

[19] J. Schwinger, “Quantum Mechanics”, Springer Verlag, 2003 ; see also G. G. Athanasiu, E. G. Floratos and S. Nicolis, J. Phys. A Math. Gen. 31, L665 (1998) and references therein.

[20] E. G. Floratos and G. K. Leontaris, Phys.Lett B412, 35 (1997) 